NASZA DERMATOLOGIA Online

OUR DERMATOLOGY Online

Source of Support:

Georgia Dermatopathology

Associates, Atlanta, Georgia, USA

Competing Interests:

None

\section{SPECIFIC CUTANEOUS HISTOLOGIC AND IMMUNOLOGIC FEATURES IN A CASE OF EARLY LUPUS ERYTHEMATOSUS SCARRING ALOPECIA}

\author{
Ana Maria Abreu Velez ${ }^{1}$, A. Deo Klein ${ }^{2}$, Michael S. Howard ${ }^{1}$ \\ ${ }^{1}$ Georgia Dermatopathology Associates, Atlanta, Georgia, USA \\ ${ }^{2}$ Statesboro Dermatology, Statesboro, Georgia, USA
}

Corresponding author: Ana Maria Abreu Velez, MD PhD

abreuvelez@yahoo.com

\begin{abstract}
Introduction: Immunoreactants detected by direct immunofluorescence (DIF) in the skin of patients with lupus erythematosus represent an important tool in the diagnosis of this disorder.

Case report: A 46 year old African American female presented complaining of hair loss and scarring in her scalp.

Methods: Biopsies for hematoxylin and eosin (H\&E) examination, as well as for direct immunofluorescence (DIF) were performed.

Results: The histologic features were representative of early lupus erythematosus. DIF demonstrated immune deposits of several immunoglobulins and complement, primarily around skin appendageal structures(hair follicles and sweat glands). Deposits of immunoglobulin $\mathrm{D}$ were seen in several areas of the epidermis.

Conclusion: In lupus erythematosus, evaluation of immune reactions against cutaneous appendageal structures may be crucial in differentiating this disorder from other autoimmune and non-autoimmune diseases.
\end{abstract}

Key words: discoid lupus erythematosus (DLE); scarring alopecia; direct immunofluorescence(DIF); skin appendices; lichen planopilaris (LPP)

Abbreviations and acronyms: Hematoxylin and eosin (H\&E), direct immunofluoresence (DIF), discoid lupus erythematosus (DLE), pseudopelade of Brocq (PB).

Ana Maria Abreu Velez, A. Deo Klein, Michael S. Howard: Specific cutaneous histologic and immunologic features in a case of early lupus erythematosus scarring alopecia. Our Dermatol Online. 2013; 4(2): 199-201

\section{Introduction}

Pseudopelade of Brocq is a progressive, scarring alopecia characterized by early alopecic patches localized in the scalp, that then coalesce into larger, irregular plaques with polycyclic borders [1]. Pseudopelade of Brocq can be considered either the final atrophic stage of multiple scarring disorders such as lichen planopilaris (LPP) and discoid lupus erythematosus (DLE), ie, secondary PB or, alternatively, a discrete nosologic disease (primary PB) [1].

\section{Case Report}

PA 46 year old African American female was evaluated for hair loss and scarring in her scalp. The patient reported a family history of lupus erythematosus. Physical examination confirmed a scarring alopecia in patches, with focal desquamation, erythema and hyperpigmentation. Skin biopsies were obtained for hematoxylin and eosin (H\&E) review, and for direct immunofluorescence. Laboratory data demonstrated a normal complete blood count (CBC) and differential analysis, and a normal erythrocyte sedimentation rate. Antiphospholipid antibody testing was negative; serum electrolytes, blood urea nitrogen, creatinine, and liver function tests, as well as urinalysis and chest radiographs were within normal limits. The antinuclear antibody (ANA) titer was normal. Specific ANA screening yielded negative results for anti-Smith, anti-double stranded DNA (dsDNA), and anti-histone antibodies. Tests for anti-ribonuclease antigen (RNAse), extractable nuclear antigen (ENA), small nuclear antigen(sn), ribonucleoproteins (RNPs), and U1 and U2 complexes were negative, as was testing for anti-SS-A (anti-Ro) and anti-SS-B (anti-La). Levels of Complement/C3 and $\mathrm{C} 4$ were within normal limits. Perinuclear anti-neutrophil cytoplasmic antibody testing was negative. However, both histologic and direct immunofluorescence (DIF) findings were representative of early lupus erythematosus. 


\section{Materials and Methods}

Hematoxylin and eosin staining was performed as previously described [2-7].

\section{Direct immunofluorescence (DIF):}

In brief, skin cryosections were prepared, and incubated with multiple fluorochromes as previously reported [2-8]. We utilized normal skin as a negative control from patients going under aesthetic plastic surgery. To test the immune response in lesional skin, we utilized the following markers: antibodies to immunoglobulins $\mathrm{A}, \mathrm{G}, \mathrm{D}, \mathrm{E}$ and $\mathrm{M}$; IgG3 and $\mathrm{IgG} 4$; Complement/C1q and $\mathrm{C} 3$; kappa light chains, lambda light chains, fibrinogen and albumin. All antibodies were fluorescein isothiocyanate (FITC) conjugated, and all obtained from Dako (Carpinteria, California, USA).

\section{Results}

Examination of the H\&E tissue sections demonstrates no significant epidermal follicular plugging. A mild, interface infiltrate of lymphocytes and histiocytes was noted. Within the dermis, a mild, superficial and deep, perivascular and periadnexal infiltrate of lymphocytes, histiocytes and plasma cells was observed. Occasional neutrophils are present within the infiltrate. Eosinophils were rare. Increased dermal mucin was not appreciated. Minimal, perifollicular dermal scarring was noted, approximating ten (10) per cent of the biopsy area. The histologic features were representative of early lupus erythematosus. The Verhoeff elastin special stain confirmed the extent of dermal scarring (Fig. 1). The PAS special stain displayed positive staining around the skin appendageal structures, and revealed no fungal organisms (Fig. 1).

Direct immunofluorescence (DIF): DIF demonstrated the following results: $\operatorname{IgG}(+$, focal granular epidermal stratum spinosum, and dermal perivascular and periadnexal); IgG3 (-); IgG4 (+, focal granular epidermal stratum spinosum, and dermal perivascular); IgA $(+$, focal granular deep dermal perivascular); IgM (+, focal granular dermal perivascular, also in superficial epidermal free nerves and periadnexal); $\operatorname{IgD}(+$, focal granular epidermal stratum spinosum cytoplasmic); IgE (-); Complement/C1q (-); Complement/C3 (+, Focal granular epidermal straum spinosum, and dermal perivascular); Kappa light chains $(++$, Focal granular epidermal stratum spinosum, and dermal perivascular and periadnexal); Lambda light chains $(+$, focal granular epidermal stratum spinosum); Albumin $(++$, focal granular dermal perivascular) and fibrinogen $(++$, focal granular epidermal stratum spinosum, and focal dermal perivascular and periadnexal). (Fig. 1). Since the H\&E biopsy demonstrated an early scarring alopecia compatible with lupus erythematosus and given the DIF results, the patient was prescribed oral prednisone, clobetasol gel, and sun protection.

\section{Discussion}

Pseudopelade of Brocq is a type of scarring alopecia of the scalp associated with a peculiar clinical presentation and evolution. Many authorities do not consider pseudopelade of Brocq a purely autonomous nosologic entity, because in $66.6 \%$ of patients it represents the end stage of other inflammatory chronic diseases such as lichen planopilaris and discoid lupus erythematosus. Primary cicatricial alopecias result from inflammatory destruction of the hair follicle, followed by its replacement by a fibrotic area [9]. It is often difficult to clinically differentiate between psueodopelade of Brocq, lichen planopilaris and discoid lupus erythematosus. Thus, histopathologic and immunopathologic studies such DIF are recommended in the workup of these disorders; overall, the appropriate diagnosis depends on clinicopathologic correlations Primary cicatricial alopecias are further subclassified as neutrophilic, lymphocytic and mixed types. Each of these groups contain specific disorders, including folliculitis decalvans, dissecting folliculitis of the scalp, erosive pustulosis of the scalp, keloidal acne of the nape, frontal fibrosing alopecia, lichen planopilaris and lupus erythematosus [9]. In our case, DIF reactivity against dermal skin appendices assisted in establishing a diagnosis of early lupus erythematosus.

Prompt diagnosis and treatment are needed in scarring lupus erythematosus to contain the hair loss, scarring and emotional distress that often accompany these sequelae [10]. The dermatologic nursing staff may facilitate the diagnostic and treatment process, and through educational and other supportive measures exert a positive impact on the patient's overall medical course [10].

\section{REFERENCES}

1. Amato L, Mei S, Massi D, Gallerani I, Fabbri P: Cicatricial alopecia; a dermatopathologic and immunopathologic study of 33 patients (pseudopelade of Brocq is not a specific clinico-pathologic entity). Int J Dermatol. 2002;41:8-15.

2. Abreu-Velez AM, Smith JG Jr, Howard MS: Activation of the signaling cascade in response to $\mathrm{T}$ lymphocyte receptor stimulation and prostanoids in a case of cutaneous lupus. N Am J Med Sci. 2011;3:251-4.

3. Abreu-Velez AM, Brown VM, Howard MS: Antibodies to piloerector muscle in a patient with lupus-lichen planus overlap syndrome. N Am J Med Sci. 2010;2:276-80.

4. Abreu-Velez AM, Girard JG, Howard MS: Antigen presenting cells in the skin of a patient with hair loss and systemic lupus erythematosus. N Am J Med Sci. 2009;1:205-10.

5. Abreu-Velez AM, Klein AD, Howard MS: Skin appendageal immune reactivity in a case of cutaneous lupus. Our Dermatol Online. 2011;2:175-80.

6.Abreu-VelezAM, Howard MS, Brzezinski P: Immunofluorescence in multiple tissues utilizing serum from a patient affected by systemic lupus erythematosus. Our Dermatol Online. 2012;3:3642.

7. Jablońska S, Chorzelski TP, Beutner EH, Michel B, Cormane R, Holubar K et al:Use of immunopathological studies in dermatology. Immunofluorescence in the diagnosis of bullous diseases, lupus erythematosus and some other diseases. Przegl Dermatol. 1976;63:267-86.

8. Abreu-Velez AM, Howard MS: Lupus: a comprehensive review. In: Lupus: Symptoms, Treatment and Potential Complications, [w] Immunology and Immune System Disorders. Thiago Devesa Marquez and Davi Urgeiro Neto (red.), AN. Nova Science Publishers, Inc. NY $11788,2011: 1-34$.

9. Piérard-Franchimont C, Piérard GE: How I explore primary cicatricial alopecias. Rev Med Liege. 2012;67:44-50.

10. Ross EK: Primary cicatricial alopecia: clinical features and management. Dermatol Nurs. 2007;19:137-43. 


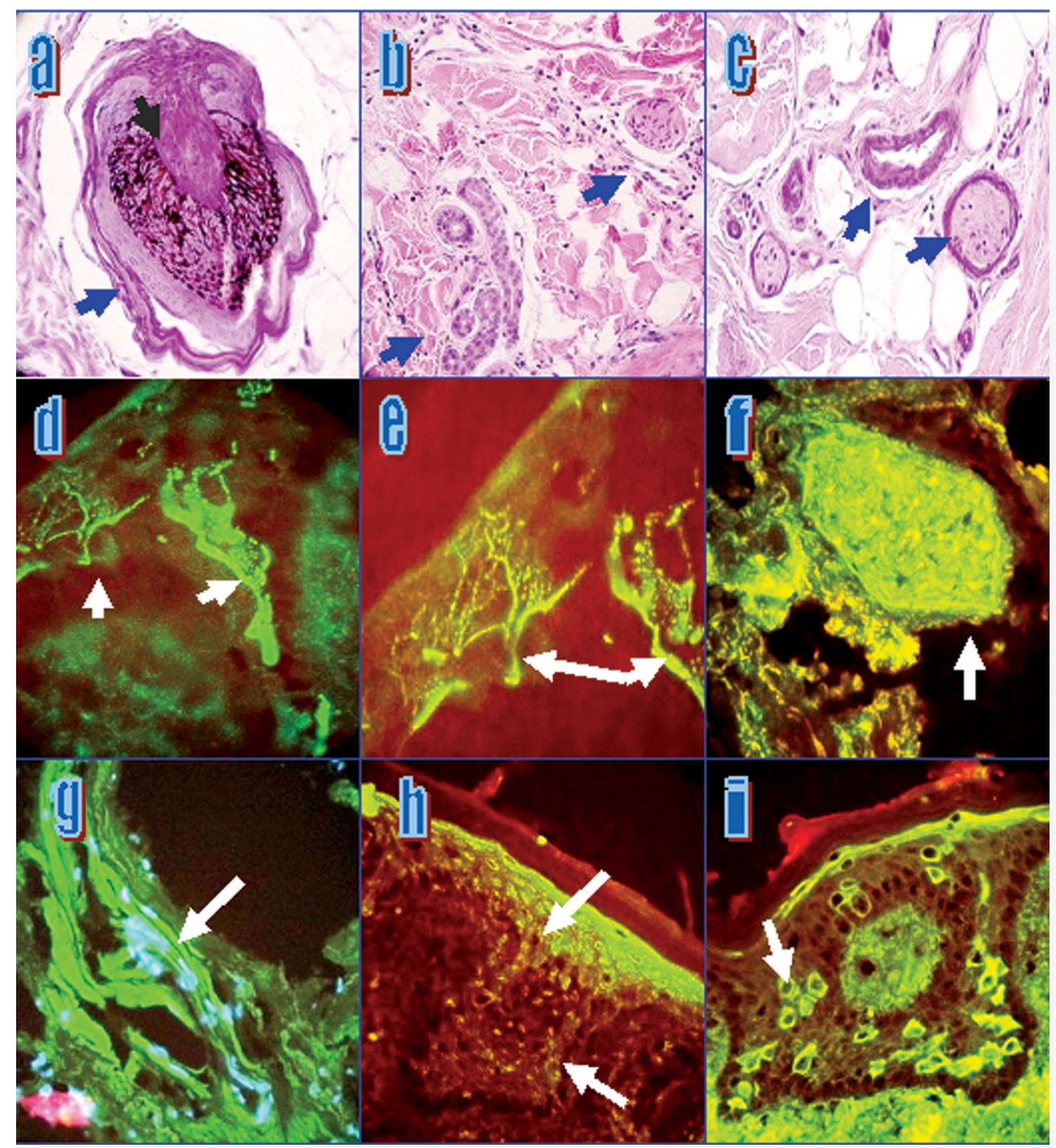

Figure 1. a PAS positive accentuation in a hair follicle outer root sheath (dark pink staining; blue arrow) and in the follicular papilla border area (green arrow) (400x). b. H\&E staining, showing a mild inflammatory infiltrate around an eccrine gland coil and a neurovascular package (blue arrows) (100x). c. PAS positive accentuation around a dermal blood vessel and nerve (200x). $\mathbf{d}$ and e. DIF using FITC conjugated anti-human IgM antibody, and demonstrating positive staining against superficial, thin nerves entering the epidermis and in the upper dermal neurovascular plexus (green staining; white arrows). f. Positive DIF staining against a sebaceous gland, utilizing FITC conjugated anti-human fibrinogen antibody (green-yellow staining; white arrow). g. Positive DIF staining against a deep dermal blood vessel, utilizing FITC conjugated anti-human lambda light chains antibody (green staining; white arrow). h. Positive DIF staining with FITC conjugated anti-human IgD antibodies against the upper and central epidermal stratum spinosum layer in an anti-nuclear and/or perinuclear keratinocytic staining pattern (white arrows). i. Positive DIF FITC conjugated anti-human lambda light chains staining, in an epidermal stratum spinosum perinuclear and pericytoplasmic pattern (white arrow).

Copyright by Ana Maria Abreu Velez, et al. This is an open access article distributed under the terms of the Creative Commons Attribution License, which permits unrestricted use, distribution, and reproduction in any medium, provided the original author and source are credited. 